\title{
Embedded surfaces in the 3-torus
}

by

\author{
Allan L. Edmonds (Bloomington, IN)
}

\begin{abstract}
Those maps of a closed surface to the three-dimensional torus that are homotopic to embeddings are characterized. Particular attention is paid to the more involved case when the surface is nonorientable.
\end{abstract}

1. Introduction. What maps of a closed, oriented surface $F_{g}$ of genus $g$ to the 3 -torus $T^{3}$ are homotopic to embeddings? While it is obvious that any orientable surface embeds, it is somewhat less clear how to characterize the maps homotopic to embeddings. Here we provide a simple, but not entirely trivial, answer to the question, as follows.

TheOREM 1.1. A map $f: F_{g} \rightarrow T^{3}$ is homotopic to an embedding if and only if one of the following conditions holds:

(1) the homology class $f_{*}\left[F_{g}\right]$ in $H_{2}\left(T^{3}\right)$ is trivial; or

(2) the homology class $f_{*}\left[F_{g}\right]$ in $H_{2}\left(T^{3}\right)$ is primitive and $f_{*}\left(H_{1}\left(F_{g}\right)\right)$ has rank 2 in $H_{1}\left(T^{3}\right)$.

Here $\left[F_{g}\right] \in H_{2}\left(F_{g}\right)$ denotes the fundamental class of the oriented surface $F_{g}$. A nontrivial element in a torsion free abelian group $G$ is said to be primitive if it is not a nontrivial multiple of another element of the group. Note also that since $T^{3}$ is aspherical, with abelian fundamental group, the set of homotopy classes of maps $X \rightarrow T^{3}$ is in one-to-one correspondence with the set of homomorphisms $H_{1}(X) \rightarrow H_{1}\left(T^{3}\right)$ for any CW complex $X$.

We also address the case of a map of a nonorientable surface $U_{h}$ (the connected sum of $h$ copies of the projective plane). Naturally the answer is a little trickier, and in many ways more interesting. The question of which nonorientable surfaces embed (with no consideration of the map) was answered by G. Bredon and J. Wood [2], who showed that $U_{h}$ embeds if and

2000 Mathematics Subject Classification: 57M99, 57R40.

Key words and phrases: 3-torus, map homotopic to embedding. 
only if $h$ is even and $h>2$. We give a complete determination of the maps homotopic to embeddings in the following.

TheOrem 1.2. A map $f: U_{h} \rightarrow T^{3}$ is homotopic to an embedding if and only if the following five conditions all hold:

(1) the nonorientable genus $h$ is even;

(2) $w_{1}\left(U_{h}\right) \in f^{*} H^{1}\left(T^{3} ; \mathbb{Z}_{2}\right)$;

(3) the mod 2 homology class $f_{*}\left[U_{h}\right]_{2}$ in $H_{2}\left(T^{3} ; \mathbb{Z}_{2}\right)$ is nonzero;

(4) the induced homomorphism $f_{*}: H_{1}\left(U_{h}\right) \rightarrow H_{1}\left(T^{3}\right)$ is surjective; and

(5) the induced integral intersection pairing on

$$
K=\left[\operatorname{ker} f_{*}: H_{1}\left(U_{h}\right) \rightarrow H_{1}\left(T^{3}\right)\right] / \text { torsion }
$$

is unimodular.

Actually, condition (2) implies (1), but not conversely. The various conditions are explained in more detail in subsequent sections. Conditions (4) and (5) are independent of each other. Condition (2) is necessary for the very definition of the intersection pairing in (5). But condition (3) is actually a consequence of the others, including (5). These latter relationships will be explained in the final section of the paper. Condition (5) may be viewed as a consequence of the fact that "the orientable part" of $U_{h}$ must also be embedded. The pairing in question is defined up to a global change of sign. The integral pairing on the kernel $K$ will be explained in more detail in Subsection 3.5.

The proofs will give a simple constructive picture of an embedded surface in each appropriate homotopy class. For an orientable surface it will be either a trivial 2-sphere with tubes attached or a standard subtorus with trivial handles attached. For any even nonorientable genus at least 4 , it will be a standard nonseparating orientable surface (the second case in Theorem 1.1), with a single nonorientable handle attached running from one side of the orientable surface to the other.

G. Bredon and J. Wood [2] treated the question of which nonorientable surfaces $U_{h}$ embed in 3-manifolds of the form $M^{2} \times S^{1}$, where $M^{2}$ is a closed orientable surface, and which homology classes in $H_{2}\left(M^{2} \times S^{1} ; \mathbb{Z}_{2}\right)$ are represented by embeddings of $U_{h}$. In particular, conditions (1)-(4) are apparent from their work. But they did not address the question of deforming a given map to an embedding.

W. Jaco [7] also studied incompressible surfaces in 3-manifolds of the form $M^{2} \times S^{1}$, where $M^{2}$ is a closed orientable surface, and in particular concluded, in the case at hand, that up to isotopy the only incompressible surfaces, orientable or not, in $T^{3}$ are standard sub-2-tori.

Alternative proofs and extensions of some of the results of Bredon and Wood have also been given by W. End [5]. 
Organization of the rest of the paper. The case of orientable surfaces is handled in Section 2 and the case of nonorientable surfaces in Section 3. The necessity of the primitivity condition in Theorem 1.1 is proved in Proposition 2.1, and the necessity of the rank 2 condition is proved in Proposition 2.3. Sufficiency in Theorem 1.1 is proved in Theorem 2.4. The necessity of conditions (1), (2), and (3) in Theorem 1.2 is proved in Corollary 3.5, Proposition 3.1, and Proposition 3.2, respectively. Condition (4) is given in Corollary 3.9. Condition (5) is explained in Proposition 3.10. Sufficiency in Theorem 1.2 is completed in Subsection 3.6. We conclude in Subsection 3.7 with examples showing the independence of conditions (4) and (5) from the other conditions in Theorem 1.2, and a proof that condition (3) follows from the other conditions.

Acknowledgements. The author thanks the referee for a careful reading of the manuscript, including pointing out an error in one proof, suggesting the alternative proof of Proposition 3.11 presented here, and recommending various improvements in exposition.

\section{Embeddings of orientable surfaces}

2.1. Primitivity of codimension one embeddings. The necessity of the primitivity condition is a well-known property of codimension one embeddings in general.

Proposition 2.1. Suppose that $N^{n}$ is a closed, oriented $n$-manifold embedded in the closed, orientable $(n+1)$-manifold $M^{n+1}$. If the homology class $\left[N^{n}\right]$ in $H_{n}\left(M^{n+1}\right)$ is nontrivial, then it is primitive, and $H_{1}\left(M^{n+1}\right) / i_{*} H_{1}\left(N^{n}\right)$ is infinite.

Proof. If $\left[N^{n}\right] \neq 0$, then $N^{n}$ must be nonseparating, and therefore taking intersection numbers with $N^{n}$ defines a homomorphism $\varphi: H_{1}\left(M^{n+1}\right) \rightarrow \mathbb{Z}$. Since $N^{n}$ must be nonseparating, there is an oriented simple closed curve $C \subset M^{n+1}$ meeting $N^{n}$ transversely exactly once, so that $C \cdot N^{n}=1$. In particular, the homomorphism $\varphi$ is surjective. On the other hand, because $N^{n}$ is orientable, it has a trivial normal bundle. From this we see that the image of $H_{1}\left(N^{n}\right)$ lies in the kernel of $\varphi$. The result follows.

2.2. Surgery on a map: the orientable case. Here we show how any map of an orientable surface to the 3 -torus is built up starting with a map of a torus or a 2-sphere. An analogous result for nonorientable surfaces will be given in Subsection 3.3.

Proposition 2.2. If $F_{g}$ is a closed orientable surface of genus $g>1$ and $f: F_{g} \rightarrow T^{3}$, then there is a nonseparating (two-sided) simple closed curve $C$ on $F_{g}$ such that the restriction $\left.f\right|_{C}$ is nullhomotopic. 
Proof. Under the present hypotheses, the homomorphism $f_{*}: H_{1}\left(F_{g}\right) \rightarrow$ $H_{1}\left(T^{3}\right)$ has a nontrivial kernel that is also primitive, just for reasons of rank. Standard facts about surfaces then imply that a primitive element in ker $f_{*}$ can be represented by the desired simple closed curve. See [1], [3], [8], and [9].

Given a simple closed curve, as in the preceding proposition, one may cut open the surface $F_{g}$ along $C$ to create a surface with two boundary components $C_{1}$ and $C_{2}$ corresponding to $C$. One may then cap off the resulting boundary components with disks $D_{1}$ and $D_{2}$ to form a closed orientable surface $F^{\prime}$ of genus $g-1$ and a map $f^{\prime}: F^{\prime} \rightarrow T^{3}$ representing the same homology class. In addition one may recover the original map $f$ up to homotopy if one records the homotopy class of a loop that the cut open handle goes around. One may do this explicitly by attaching to $F^{\prime}$ an embedded $\operatorname{arc} A$ with both end points at the centers of the disks $D_{1}$ and $D_{2}$ with $f^{\prime}$ extended over the arc $A$ as dictated by the original map $f$. Clearly, one can recover $f$ from the extended $f^{\prime}$ by running a tube along the path $f^{\prime}(A)$.

One may also iterate this process, eventually reducing to a torus with $g-1$ arcs attached. Either the map of the torus is injective on homology or one can surger one more time to obtain a 2 -sphere.

2.3. Embedded surgery: the orientable case. Here we prove the necessity of the rank condition on the image of $H_{1}$.

Proposition 2.3. Let $f: F_{g} \rightarrow T^{3}$ be an embedding of a closed, orientable surface of genus $g$ that represents a primitive homology class $f_{*}\left[F_{g}\right]$ in $H_{2}\left(T^{3}\right)$. Then the image $f_{*} H_{1}\left(F_{g}\right)$ in $H_{1}\left(T^{3}\right)$ is isomorphic to $\mathbb{Z} \oplus \mathbb{Z}$ and is a summand of $H_{1}\left(T^{3}\right)$.

Proof. By Proposition 2.1 the rank of the image cannot be 3 . If the rank were 0 then the embedding $f$ lifts to an embedding in the universal covering $\mathbb{R}^{3}$, which would imply that $f_{*}\left[F_{g}\right]=0$. Similarly, if the rank of the image $f_{*} H_{1}\left(F_{g}\right)$ were 1 , then $f$ would lift to a covering homeomorphic to $S^{1} \times \mathbb{R}$, again implying that $f_{*}$ is trivial on $H_{2}$ and contradicting our standing assumption here. Finally, if $f_{*} H_{1}\left(F_{g}\right)$ has rank 2 but is not a summand, then $f$ would lift to a covering of the form $T^{2} \times \mathbb{R}$ for which the projection to $T^{3}$ maps $H_{1}\left(T^{2} \times \mathbb{R}\right)$ to a nonprimitive subgroup of $H_{1}\left(T^{3}\right)$. But such a map takes $H_{2}\left(T^{2} \times \mathbb{R}\right)$ to a nonprimitive subgroup of $H_{2}\left(T^{3}\right)$, contradicting the assumed primitivity of $f_{*} H_{2}\left(F_{g}\right)$.

2.4. Embedding theorem: orientable case. Here we present the main embedding theorem in the case of a map of a general closed, orientable surface.

THEOREM 2.4. Let $f: F_{g} \rightarrow T^{3}$ be a map of a genus $g$ surface $(g \geq 1)$ to the torus $T^{3}$, such that the homology class $f_{*}\left[F_{g}\right]$ in $H_{2}\left(T^{3}\right)$ vanishes or 
the homology class $f_{*}\left[F_{g}\right]$ in $H_{2}\left(T^{3}\right)$ is primitive and $f_{*} H_{1}\left(F_{g}\right)$ in $H_{1}\left(T^{3}\right)$ has rank two (not more). Then $f$ is homotopic to an embedding.

Proof. In the first case the surgery-on-a-map process of Subsection 2.2 reduces $f$ to a map $f^{\prime}: S^{2} \cup_{g} A_{i} \rightarrow T^{3}$, of a 2-sphere union a collection of $g$ arcs. The 2-sphere is nullhomotopic and so $\left.f^{\prime}\right|_{S^{2}}$ may be assumed to be an embedding onto a standard 2-sphere bounding a 3-ball. By general position it may further be assumed that $f^{\prime}$ embeds all the arcs and indeed that $f^{\prime}$ is an embedding overall. Finally, one can add tubes to $S^{2}$ along these embedded arcs to produce the desired embedding homotopic to $f$.

In the second case the surgery-on-a-map process reduces the given map $f$ to a map $f^{\prime}: T^{2} \cup_{g-1} A_{i} \rightarrow T^{3}$, of a 2-torus union a collection of $g-1$ arcs. The map $f^{\prime}$ on the torus is homologically nontrivial and primitive, and by Proposition 2.3 it may be assumed that $\left.f^{\prime}\right|_{T^{2}}$ is an embedding onto a standard subtorus $T^{2}$ of $T^{3}$. Moreover, $f^{\prime}$ is homotopic to a map taking the $\operatorname{arcs} A_{i}$ into $T^{2}$ as well, by the condition on the rank of $f_{*} H_{1}\left(F_{g}\right)$. By a further homotopy one can assume the arcs are embedded in $T^{2}$. Finally, one can perturb the map on these arcs so that they are small, trivially embedded arcs on one side of $T^{2}$.

Now one can add small tubes along the arcs $A_{i}$, extending the map $f^{\prime}$ appropriately to produce the desired embedding.

\section{Embeddings of nonorientable surfaces}

3.1. General nonorientability issues. It is, of course, not true that a codimension-one submanifold of an orientable manifold must be orientable, as one knows from the simple example of $\mathbb{R} P^{2} \subset \mathbb{R} P^{3}$.

The Stiefel-Whitney class. We think of the first Stiefel-Whitney class as a homomorphism $w_{1}: H_{1}\left(N^{n}\right) \rightarrow \mathbb{Z}_{2}=\{0,1\}$, where $w_{1}(\lambda)=1$ if and only if a local orientation of $N^{n}$, when transported once around a loop representing $\lambda$, is reversed. We also view $w_{1}\left(N^{n}\right)$ as an element of $H^{1}\left(N^{n} ; \mathbb{Z}_{2}\right)$. More properly, this is $w_{1}\left(T N^{n}\right)$. We may similarly speak of $w_{1}(\xi)$ for any vector bundle over $N^{n}$.

Proposition 3.1. Suppose that $N^{n}$ is a closed $n$-manifold embedded in the orientable $(n+1)$-manifold $W^{n+1}$ with normal bundle $\nu$. Then $w_{1}\left(N^{n}\right)=$ $w_{1}(\nu)=\varphi \mid N^{n}$ for some $\varphi \in H^{1}\left(W^{n+1} ; \mathbb{Z}_{2}\right)$.

Proof. Define $\varphi \in H^{1}\left(W^{n+1} ; \mathbb{Z}_{2}\right)=\operatorname{Hom}\left(H_{1}\left(W^{n+1}\right), \mathbb{Z}_{2}\right)$ by $\bmod 2$ intersection numbers of 1-cycles with the submanifold $N^{n}$. The result follows since a loop in $N^{n}$ is orientation-reversing in $N^{n}$, if and only if it is orientation-reversing in $\nu$, if and only if it has mod 2 intersection number 1 with $N^{n}$. 
See also Milnor-Stasheff [10, pp. 39 and 119], for example. In particular, this implies the well-known fact that a closed nonorientable surface cannot embed in $\mathbb{R}^{3}$.

Proposition 3.2. Suppose that $N^{n}$ is a closed nonorientable $n$-manifold embedded in the closed, orientable $(n+1)$-manifold $M^{n+1}$. Then $\left[N^{n}\right]_{2}$ is nontrivial in $H_{n}\left(M^{n+1} ; \mathbb{Z}_{2}\right)$.

Proof. If $\left[N^{n}\right]_{2}=0$, then $N^{n}$ separates $M^{n+1}$. But that shows that $N^{n}$ has a trivial normal bundle. And that in turn would imply that $N^{n}$ is orientable.

The Poincaré dual $D w_{1}\left(N^{n}\right)$ lies in $H_{n-1}\left(N^{n} ; \mathbb{Z}_{2}\right)$ and can be represented by an embedded $(n-1)$-dimensional submanifold, in general, as the transverse pre-image of $\mathbb{R} P^{k-1}$ under a map $f: N^{n} \rightarrow \mathbb{R} P^{k}$ representing $w_{1}\left(N^{n}\right)$, where $\mathbb{R} P^{\infty} \simeq K\left(\mathbb{Z}_{2}, 1\right)$.

If $F=U_{h}$, the connected sum of $h$ copies of the projective plane, then we can identify $D w_{1}(F)$ concretely as being represented by the disjoint union of the corresponding $h$ copies of $\mathbb{R} P^{1}$. It has the defining property that its complement in $F$ is orientable and the complement of no proper subcollection is orientable. With proper orientations on these circles, twice the integral class represented by the union is 0 in $H_{1}(F)$.

On the other hand, one can calculate directly that $H_{1}\left(U_{h}\right) \approx \mathbb{Z}^{h-1} \oplus \mathbb{Z}_{2}$. It follows that the unique element of order 2 represents the Poincaré dual of $w_{1}$ as an element of $H_{1}\left(U_{h}\right)$.

Although $D w_{1}\left(N^{n}\right)$ is represented by an integral homology class, $w_{1}\left(N^{n}\right)$ itself is not always represented by an integral cohomology class.

Lemma 3.3. The first Stiefel-Whitney class $w_{1}\left(U_{h}\right)$ is the mod 2 reduction of an integral cohomology class if and only if $h$ is even.

Proof. The cohomology class $w_{1}$ when viewed as a homomorphism $\varphi$ : $H_{1}\left(U_{h}\right) \rightarrow \mathbb{Z}_{2}$ is characterized by $\varphi(x)=x \cdot x \bmod 2$. Such a homomorphism is the mod 2 reduction of a homomorphism $H_{1}\left(U_{h}\right) \rightarrow \mathbb{Z}$ if and only if $\varphi\left(D w_{1}\left(U_{h}\right)\right)=0$, if and only if the element of order 2 in $H_{1}\left(U_{h}\right)$ is orientation-preserving, if and only if $h$ is even.

3.2. Consequences for nonorientable surfaces in the 3-torus. Because all mod 2 cohomology classes of the torus are reductions of integral classes, we have the following consequence of Proposition 3.1.

COROLlary 3.4. If a closed surface $F$ embeds in $T^{3}$ then $w_{1}(F)$ is the mod 2 reduction of an integral cohomology class.

Since the first Stiefel-Whitney class of a surface of odd genus $h$ is not the reduction of an integral cohomology class, the following result is immediate. 
COROLlary 3.5. If a closed nonorientable surface $U_{h}$ embeds in the 3 -torus then it has even genus $h$.

Working a little harder with cohomology rings we can deduce the following, known, result.

Proposition 3.6 (Bredon and Wood [2, (4.7)], Jaco [7, Cor. 5.3]). A Klein bottle does not embed in $T^{3}$.

Proof. We offer a proof valid for any 3-manifold with the integral cohomology of $T^{3}$. Suppose a Klein bottle $K^{2} \subset T^{3}$. By Proposition 3.2, $H_{2}\left(K^{2} ; \mathbb{Z}_{2}\right) \rightarrow H_{2}\left(T^{3} ; \mathbb{Z}_{2}\right)$ is nontrivial. Dually the cohomology mod 2 fundamental class must lie in the image of $H^{2}\left(T^{3} ; \mathbb{Z}_{2}\right)$.

Now the integral and mod 2 cohomology rings of $T^{3}$ are generated by three elements of degree 1 with their squares equal to 0 . Mod 2 all squares vanish.

As for the Klein bottle, its mod 2 cohomology ring is generated by two elements of degree 1 with nonvanishing squares, whose product is trivial, as one can see by viewing $K^{2}$ as the connected sum of two projective planes.

Now, the mod 2 cohomology generators of the torus are reductions of integral classes. But only one of the three nonzero elements of the mod 2 cohomology of the Klein bottle is integral. It follows that the image of $H^{1}\left(T^{3} ; \mathbb{Z}_{2}\right) \rightarrow H^{1}\left(K^{2} ; \mathbb{Z}_{2}\right)$ has rank at most one, and hence that $H^{2}\left(T^{3} ; \mathbb{Z}_{2}\right)$ $\rightarrow H^{2}\left(K^{2} ; \mathbb{Z}_{2}\right)$ vanishes. This contradicts the fact that $H_{2}\left(K^{2} ; \mathbb{Z}_{2}\right) \rightarrow$ $H_{2}\left(T^{3} ; \mathbb{Z}_{2}\right)$ is nontrivial and therefore implies that $K^{2}$ does not embed in $T^{3}$ after all.

REMARK 1. Here is yet another argument for the preceding result, this time, taking more advantage of the structure of the standard torus. If $f$ : $U_{2} \rightarrow T^{3}$ is an embedding, then the image subgroup $f_{*}\left(\pi_{1}\left(U_{2}\right)\right) \subset \pi_{1}\left(T^{3}\right)$ must be cyclic, as one sees by abelianizing. Thus such a map $f$ lifts to an embedding in a covering space of $T^{3}$ of the form $S^{1} \times \mathbb{R}^{2}$. The latter embeds in $\mathbb{R}^{3}$. But it is standard that closed, nonorientable surfaces do not embed in $\mathbb{R}^{3}$.

REMARK 2. It is crucial that the 3-manifold in the previous result have the cohomology ring structure of the torus. The manifold $M^{3}=S^{1} \times S^{2} \#$ $S^{1} \times S^{2} \# S^{1} \times S^{2}$ has the integral homology groups of $T^{3}$. But the Klein bottle embeds in $S^{1} \times S^{2}$, hence in $M^{3}$, as one can see by adding a handle to a standard $S^{2}$ that goes once around in the $S^{1}$ direction.

3.3. Surgery on a map. There is a simple statement about surgery on a map of a nonorientable surface when the nonorientable genus is even. We give a formulation that applies to most nonorientable surfaces of odd genus as well. 
Proposition 3.7. If $U_{h}$ is a closed nonorientable surface, $h \neq 1,3$, and $f: U_{h} \rightarrow T^{3}$, then there is a nonseparating, two-sided simple closed curve $C$ on $U_{h}$ such that $\left.f\right|_{C}$ is nullhomotopic.

Proof. If $h$ is odd we may write $U_{h}=F_{g} \# U_{1}$, where $h=2 g+1$. If $h$ is even, then we may write $U_{h}=F_{g} \# U_{2}$, where $h=2 g+2$. Assuming $h \geq 4$, we get $g \geq 2$. It follows as in the orientable case that the desired simple closed curve exists, lying in an orientable part of $U_{h}$.

If $h$ is even, including the case when $h=2$, let $C \subset U_{h}$ be a simple closed curve that represents the element of order 2 in $H_{1}\left(U_{h}\right) \approx \mathbb{Z}^{h-1} \oplus \mathbb{Z}_{2}$. Since $h$ is even, $C$ is 2 -sided. Since $\pi_{1}\left(T^{3}\right)=H_{1}\left(T^{3}\right)$ is torsion-free, it follows that $\left.f\right|_{C}$ is nullhomotopic as required.

3.4. Embedded surgery. It is well known that a one-sided incompressible surface need not be $\pi_{1}$-injective in general. But the conditions of incompressibility and $\pi_{1}$-injectivity turn out to be equivalent for surfaces in $T^{3}$ and other 3-manifolds that are products with a circle, by results of Jaco [7].

Proposition 3.8 (see [7, Theorem 5.2]). Let $U_{h} \subset T^{3}$ be an embedded, closed, nonorientable surface of even genus $h$. Then $U_{h}$ is compressible; that is, there is a homotopically nontrivial, two-sided, simple closed curve $C \subset U_{h}$ such that $C$ bounds an embedded disk $D \subset T^{3}$ such that $D \cap U_{h}=C$.

Proof discussion. For the convenience of the reader we sketch the proof with some attention to the orientability issues and the fact that the containing manifold is a torus. The proof shows that up to isotopy the only incompressible surfaces in $T^{3}$ are standard 2-tori.

Consider an arbitrary connected, closed surface $M^{2} \subset T^{3}$. Choose any standard 2-torus $T^{2} \subset T^{3}$. Isotope $M^{2}$ so that it intersects $T^{2}$ transversely with the minimum possible number of components of intersection.

If the intersection $M^{2} \cap T^{2}$ were empty, then $M^{2}$ would embed in $T^{3}$ $T^{2} \cong T^{2} \times \mathbb{R}$, which embeds in $\mathbb{R}^{3}$, which means $M^{2}$ would be orientable. Moreover, it would be compressible, unless $M^{2}$ were a $\pi_{1}$-injective torus, in $T^{2} \times \mathbb{R}$. See Hempel [6, Lemma 6.1], for example. And in this case it would be parallel to the standard $T^{2}$.

Therefore we may assume that the intersection consists of a nonempty collection of pairwise disjoint simple closed curves. Among these there cannot be any curves nullhomotopic in both $T^{2}$ and $M^{2}$. Indeed, an innermost such curve on the torus $T^{2}$ bounds a disk on the torus and also bounds a disk on $M^{2}$. The 2-sphere comprised of these two disks bounds a 3-ball in $T^{3}$, so that one could eliminate at least one curve of intersection by an isotopy, contradicting minimality.

If among these curves there are ones that are nullhomotopic in $T^{2}$, but not in $M^{2}$, then choosing such a curve that is innermost in $T^{2}$, we obtain 
the desired compressing disk. Otherwise, $M^{2} \cap T^{2}$ must consist of a finite number of parallel, homotopically nontrivial, simple closed curves on the torus. We will see that in this case $M^{2}$ must also be a torus.

Cutting open $T^{3}$ and $M^{2}$ along this $T^{2}$ we obtain an incompressible surface $M_{1}^{2}$ in $T^{2} \times I$, with nonempty boundary contained in $T^{2} \times\{0,1\}$, with the additional property that the curves of $\partial M_{1}^{2}$ in $T^{2} \times\{0\}$ and $T^{2} \times\{1\}$ have the same slope.

One may argue that up to isotopy such a surface must consist of a family of parallel vertical annuli. Compare Waldhausen [11, 3.1], and Jaco [7, 5.1]. (The case of a boundary-parallel surface does not occur, since otherwise it could be used to reduce the number of components of the intersection of $M^{2}$ with $T^{2}$.) For us these references are slightly inadequate, since Jaco at the crucial point refers the reader to Waldhausen, and Waldhausen explicitly considers only orientable surfaces. We therefore offer a few more details.

One may assume, after composing with a diffeomorphism of $T^{2}$, that the components of $M_{1}^{2} \cap T^{2} \times\{0,1\}$ are all of the form $S^{1} \times\{x\} \times\{\varepsilon\}, x \in S^{1}$ and $\varepsilon \in\{0,1\}$. One may then choose an annulus $A=S^{1} \times\{y\} \times I$ with $\partial A$ disjoint from $M_{1}^{2}$.

Make $M_{1}^{2}$ transverse to $A$, meeting $A$ in a minimal number of simple closed curves. In particular, we may assume that $M_{1}^{2} \cap A$ contains no simple closed curves that are trivial on $A$ or on $M_{1}$, and so that all components of the intersection are parallel on $A$. Then cut $T^{2} \times I$ and $M_{1}^{2}$ open along $A$, obtaining a solid torus $S^{1} \times I^{2}$ containing an incompressible surface $M_{2}^{2}$. This incompressible surface has the property that its boundary curves consist of parallel longitudes on $S^{1} \times I^{2}$.

This surface $M_{2}^{2}$ must consist of boundary-parallel annuli. To see this consider how $M_{2}^{2}$ intersects a meridian disk $D^{2} \subset S^{1} \times I^{2}$. The intersection may be assumed to be transverse. Since $M_{2}^{2}$ is incompressible, one may eliminate any circles of intersection. Thus we have a system of properly embedded arcs in $D^{2}$. Now consider an outermost arc of intersection of $M_{2}^{2} \cap D^{2}$. A boundary compression, using the disk spanned by this outermost arc and the corresponding arc on the boundary of $D^{2}$, produces an incompressible surface with a boundary component that is a trivial simple closed curve in the boundary of the solid torus. Incompressibility implies that this simple closed curve is the boundary of a disk in the boundary compressed surface. Such a disk is boundary-parallel in $S^{1} \times I^{2}$. We conclude that the corresponding component of $M_{2}^{2}$ is a boundary-parallel annulus.

Gluing things back together to form $T^{2} \times I$ and then gluing the ends of $T^{2} \times I$ back together displays $M^{2}$ as a union of annuli identified along their boundary curves. We already know that $M^{2}$ cannot be a Klein bottle. We conclude that $M^{2}$ must in fact be a torus. In fact, it is displayed as a 
covering of a standard subtorus. Primitivity of embeddings then shows that $M^{2}$ maps by degree 1 to this subtorus and is thus isotopic to the standard subtorus. In particular, at this point in the argument $M^{2}$ must be orientable.

Thus, when $M^{2}$ is nonorientable, we will always find a compressing disk in $T^{2}$ with boundary an innermost nullhomotopic curve of intersection.

Corollary 3.9. Let $U_{h} \subset T^{3}$ be an embedded, closed, nonorientable surface of genus $h$. Then the inclusion-induced homomorphism $H_{1}\left(U_{h}\right) \rightarrow$ $H_{1}\left(T^{3}\right)$ is surjective.

Proof. Let $C \subset U_{h}$ be a nontrivial simple closed curve that bounds an embedded disk $D$ such that $D \cap U_{h}=C$, and let the closed surface obtained by surgering $U_{h}$ along $C$ be denoted by $F$.

If $F$ is nonorientable ( $F$ might have two components in this case), then an inductive argument implies that $H_{1} F \rightarrow H_{1} T^{3}$ is onto. It follows that $H_{1}\left(U_{h}\right) \rightarrow H_{1}\left(T^{3}\right)$ is onto, as required.

Now suppose that $F$ is orientable. Then $C$ represents $D w_{1}\left(U_{h}\right)$, and, since $F$ must be homologically nontrivial as $U_{h}$ is mod 2 homologically nontrivial, the previous analysis of the orientable case implies that the image of $H_{1}(F)$ in $H_{1}\left(T^{3}\right)$ is a rank 2 summand. Then $U_{h}$ is obtained from $F$ by running a tube along an embedded path that runs exactly once from one side of $F$ to the other. It follows that the image of $H_{1}\left(U_{h}\right)$ is all of $H_{1}\left(T^{3}\right)$.

REMARK 3. The preceding corollary is also a consequence of the fundamental group calculations of Bredon and Wood [2, (4.4)].

3.5. The induced pairing on the kernel. The full intersection pairing on the integral first homology of a nonorientable surface is only well-defined mod 2. However, if $A$ and $B$ are oriented, orientation-preserving closed curves in a nonorientable surface $M^{2}$, they have a well-defined integral intersection number up to sign. We assume the curves are regularly immersed and intersect transversely. The normal bundle of $A$, say, can be oriented. Then each point of intersection of $B$ with $A$ can be given a sign and these signs may be added up. Of course, reversing the orientation of the normal bundle or reversing the orientation of one of the two curves will reverse the signs. Changing $A$ or $B$ by a homology preserves the absolute value of the net algebraic intersection number.

If all curves in question lie in an open, connected, orientable subset $V \subset M^{2}$, then we may orient $V$ and use that orientation to orient the normal bundles of all immersed curves in $V$. Thus an orientation on $V$ gives a well-defined skew-symmetric integer-valued pairing on $H_{1}(V)$, providing a definite sign to the intersection number $A \cdot B$ described above. 
Now suppose that $f: U_{h} \rightarrow T^{3}$ is any map that induces a surjection on integral homology and such that $w_{1}\left(U_{h}\right) \in f^{*} H^{1}\left(T^{3} ; \mathbb{Z}_{2}\right)$. Set

$$
K=\left[\operatorname{ker} f_{*}: H_{1}\left(U_{h}\right) \rightarrow H_{1}\left(T^{3}\right)\right] / \text { torsion. }
$$

According to the preceding results and earlier results about the first StiefelWhitney class, we know that $K$ is a free abelian group of even rank $h-4$ on which the first Stiefel-Whitney class, viewed as a homomorphism on the first homology group, vanishes. Moreover, the preceding remarks can be used to show that $K$ admits an integer-valued intersection pairing that is well-defined up to a global change of sign, by finding an orientable subsurface carrying all of $K$ at once, as we now explain.

The mod 2 reduction of the unique element of order 2 in $H_{1}\left(U_{h}\right)$ is the Poincaré dual $D w_{1}\left(U_{h}\right)$ and is represented by a two-sided simple closed curve $C \subset U_{h}$. Let $F_{0}$ denote the result $U_{h}-\operatorname{int} N(C)$ of cutting $U_{h}$ open along $C$ and $F_{0}^{\prime}$ denote the result of capping off the resulting two boundary components with disks. Let $f_{0}: F_{0} \rightarrow T^{3}$ denote the restriction of $f$ to $F_{0}$. We can extend $f_{0}$ to a map $f_{0}^{\prime}: F_{0}^{\prime} \rightarrow T^{3}$ since the boundary curves of $F_{0}$ represent the same class as $C$ itself, which maps nullhomotopically to $T^{3}$, because $C$ has order 2 in homology while $H_{1}\left(T^{3}\right)$ is torsion free. The extension is unique up to homotopy since $\pi_{2}\left(T^{3}\right)=0$. Both $F_{0}$ and $F_{0}^{\prime}$ are orientable and connected, and we choose compatible orientations for both.

Let

$$
\begin{aligned}
K_{0} & =\left[\operatorname{ker} f_{0 *}: H_{1}\left(F_{0}\right) \rightarrow H_{1}\left(T^{3}\right)\right] / \text { boundary classes, } \\
K_{0}^{\prime} & =\left[\operatorname{ker} f_{0 *}^{\prime}: H_{1}\left(F_{0}^{\prime}\right) \rightarrow H_{1}\left(T^{3}\right)\right] .
\end{aligned}
$$

If a class $\gamma \in \operatorname{ker} f_{*}$, then $\gamma \cdot C=0$ (not just mod 2), since $\gamma$ and $C$ are both orientation-preserving, but $C$ represents a torsion element in homology. Thus such a $\gamma \in \operatorname{ker} f_{*}$ can be represented by an element of $H_{1}\left(F_{0}\right)$ (say by surgering pairs of intersections with $C$ of opposite sign, of a curve representing $\gamma$ ).

It follows that inclusion induces isomorphisms

$$
K_{0} \rightarrow K \quad \text { and } \quad K_{0} \rightarrow K_{0}^{\prime} .
$$

The desired pairing, then, is given by the restriction of the nonsingular, skew-symmetric intersection pairing on the closed oriented surface $F_{0}^{\prime}$.

We will say that the kernel $K$, together with its intersection pairing, is carried by the orientable subsurface $F_{0} \subset U_{h}$, as well as by the capped-off surface $F_{0}^{\prime}$. The determinant of this skew-symmetric bilinear form space, a nonnegative integer, is an invariant of the embedding. We will say that the kernel $K$ is unimodular if this determinant is 1 .

The preceding discussion shows that the isomorphism type of the intersection pairing on the kernel (mod torsion) $K$, and in particular its determinant, are well-defined, depending only on the original map $f$ and not the 
choice of the simple closed curve $C$ representing the element of order 2 in homology.

Proposition 3.10. If $f: U_{h} \rightarrow T^{3}$ is an embedding, then the intersection pairing on $K=\left[\operatorname{ker} f_{*}: H_{1}\left(U_{h}\right) \rightarrow H_{1}\left(T^{3}\right)\right] /$ torsion is unimodular.

Proof. Note that the rank of $K$ is $(h-1)-3=h-4$, which is even. As above, let $C \subset U_{h}$ be a nontrivial two-sided simple closed curve that bounds an embedded disk $D$ such that $D \cap U_{h}=C$, and let the surface $F$ be obtained by surgering $U_{h}$ along $C$.

First suppose that $F$ is orientable. Then $F$ must be homologically nontrivial as $U_{h}$ is mod 2 homologically nontrivial. The previous analysis of homologically nontrivial orientable surfaces implies that the image of $H_{1}(F)$ in $H_{1}\left(T^{3}\right)$ is a rank 2 summand, which $F$ maps to by degree \pm 1 . Then we see that $K=\left[\operatorname{ker} f_{*}: H_{1}\left(U_{h}\right) \rightarrow H_{1}\left(T^{3}\right)\right] /$ torsion $=\operatorname{ker} f_{*}: H_{1}(F) \rightarrow H_{1}\left(T^{2}\right)$. The latter is unimodular as a consequence of the ordinary Poincaré duality, as in the Wall surgery theory, as required. More geometrically, we can view $f^{\prime}$ as a degree 1 map $F \rightarrow T^{2}$. So by results of Edmonds (see [4]), $f^{\prime}$ is homotopic to a pinch. The pinched portion represents $K$ as the homology of a closed orientable surface, hence admitting a unimodular intersection pairing.

Now suppose that $F$ is nonorientable. First suppose $F$ is also connected. Then an inductive argument implies that $K^{\prime}=\left[\operatorname{ker} f_{*}^{\prime}: H_{1}(F) \rightarrow\right.$ $\left.H_{1}\left(T^{3}\right)\right] /$ torsion is unimodular of rank $(h-2)-4=h-6$. Moreover, $f_{*}^{\prime}: H_{1}(F) \rightarrow H_{1}\left(T^{3}\right)$ is surjective. It follows that the surgery curve $C$ contributes to the kernel as does a suitable dual class killed by the surgery (since its image also comes from $F$ ). This adds a unimodular rank 2 summand to $K^{\prime}$, showing that $K$ also is unimodular. The addition is not necessarily quite orthogonal:

$$
\left[\begin{array}{ccccc} 
& & & 0 & * \\
& K^{\prime} & & \vdots & \vdots \\
& & & 0 & * \\
0 & \ldots & 0 & 0 & 1 \\
* & \ldots & * & -1 & 0
\end{array}\right]
$$

But simultaneous row and column operations, adding multiples of $C$ to basis elements of $K^{\prime}$, convert the sum into an orthogonal sum, of kernels, from which it is clear that the result is unimodular.

It remains to consider the case when $F$ is nonorientable and consists of two components. It is impossible that both components are nonorientable, as we show in Proposition 3.11 below. 
Therefore we may suppose that $F$ consists of one component $F^{\prime}$ that is nonorientable and one component $F^{\prime \prime}$ that is orientable. We apply induction to the nonorientable component to conclude that $K^{\prime}=\left[\operatorname{ker} f_{*}^{\prime}: H_{1}\left(F^{\prime}\right) \rightarrow\right.$ $\left.H_{1}\left(T^{3}\right)\right] /$ torsion is unimodular. Again $f_{*}^{\prime}: H_{1}\left(F^{\prime}\right) \rightarrow H_{1}\left(T^{3}\right)$ is surjective. We need to argue that the homology of the orientable component $F^{\prime \prime}$ adds an appropriate unimodular summand, showing that $K$ also is unimodular. This time the sum is clearly an orthogonal sum. What we need to see is that the entire homology of the orientable part $F^{\prime \prime}$ contributes to the kernel. Because $F^{\prime \prime}$ is nullhomologous by Proposition 3.11, elementary considerations of duality show that half a symplectic basis for $H_{1}\left(F^{\prime \prime}\right)$ lies in the kernel. Because $f_{*}^{\prime}: H_{1}\left(F^{\prime}\right) \rightarrow H_{1}\left(T^{3}\right)$ is surjective we may alter the other half of a symplectic basis for $F^{\prime \prime}$ by adding appropriate elements from the $H_{1}\left(F^{\prime}\right)$. This makes $K^{\prime \prime}=H_{1}\left(F^{\prime \prime}\right)$, but now the direct sum $K^{\prime} \oplus K^{\prime \prime}$ is not entirely orthogonal. Finally, we may alter basis elements of $K^{\prime}$ by adding multiples of the first half of the symplectic basis for $K^{\prime \prime}$ to make the sum orthogonal. The result follows.

Proposition 3.11. Let $N_{1}$ and $N_{2}$ be disjoint, closed, connected, $n$ dimensional submanifolds in a closed, connected, orientable $(n+1)$-manifold $M$, with $\pi_{1}(M)$ abelian. Then at least one of the submanifolds is orientable. If only one is orientable, then it represents a trivial homology class in $H_{n}(M)$. If both are orientable and nontrivial in $H_{n}(M)$, then they represent the same homology class up to sign.

Proof. Given a pair of submanifolds $N_{1}$ and $N_{2}$ in $M$, define a homomorphism

$$
\varphi: \pi_{1}\left(M, x_{0}\right) \rightarrow \mathbb{Z}_{2} * \mathbb{Z}_{2}
$$

as follows. Represent an element of $\pi_{1}\left(M, x_{0}\right)$ by a loop $\alpha:(I,\{0,1\}) \rightarrow$ $\left(M, x_{0}\right)$, perturbed to meet the submanifolds transversely in isolated points. Starting at the base point read off a word in two symbols, $x_{1}$ corresponding to $N_{1}$ and $x_{2}$ corresponding to $N_{2}$, as one intersects the surfaces, in succession, around the loop. Then view the two symbols as the canonical generators of the two factors of $\mathbb{Z}_{2} * \mathbb{Z}_{2}$, and map the homotopy class of the loop to the corresponding word in these two generators. This is easily seen to describe a well-defined homomorphism.

If both submanifolds are nonorientable, hence nonseparating, then it is clear that this homomorphism $\varphi$ is surjective, contradicting the assumption that $\pi_{1}\left(M, x_{0}\right)$ is abelian. More generally, the same contradiction arises for any pair of codimension-one submanifolds $N_{1}$ and $N_{2}$ such that $M-N_{1} \cup N_{2}$ is connected. From this the second and third conclusions also follow.

3.6. Embedding theorem. Finally, we extend the orientable embedding theorem to the case of a map of a general closed, nonorientable surface $U_{h}$ of nonorientable genus $h$. 
THEOREM 3.12. Let $f: U_{h} \rightarrow T^{3}$ be a map of a nonorientable surface of genus $h$ to the torus $T^{3}$ such that the following conditions all hold:

(1) the nonorientable genus $h$ is even;

(2) $w_{1}\left(U_{h}\right) \in f^{*} H^{1}\left(T^{3} ; \mathbb{Z}_{2}\right)$;

(3) the mod 2 homology class $f_{*}\left[U_{h}\right]_{2}$ in $H_{2}\left(T^{3} ; \mathbb{Z}_{2}\right)$ is nonzero;

(4) the induced homomorphism $f_{*}: H_{1}\left(U_{h}\right) \rightarrow H_{1}\left(T^{3}\right)$ is surjective;

(5) the induced integral intersection pairing on is unimodular.

$$
K=\left[\operatorname{ker} f_{*}: H_{1}\left(U_{h} \rightarrow H_{1}\left(T^{3}\right)\right] /\right. \text { torsion }
$$

Then $f$ is homotopic to an embedding.

Proof. We will proceed by induction on the even number $h$. The base case is $h=4$, which we will address after some preliminary work applicable to both the base case and the inductive case.

We may choose a product structure on $T^{3}=T^{2} \times S^{1}$ such that the homdual $\zeta$ of $z=\left[\left(x_{0}, y_{0}\right) \times S^{1}\right]$ pulls back to represent $w_{1}\left(U_{h}\right)$ after reduction $\bmod 2$. Let $\pi: T^{3} \rightarrow S^{1}$ denote the projection on the last factor. It follows that the composition $\pi f: U_{h} \rightarrow S^{1}$ is surjective on $\pi_{1}$. By transversality and primitivity we may assume that $(\pi f)^{-1}$ (point) is a single two-sided simple closed curve $C$, which necessarily represents $D w_{1}\left(U_{h}\right)$.

Surgery on the map $f$ along this two-sided simple closed curve $C$ yields a map $f^{\prime}: F_{g} \rightarrow T^{3}$, where $F_{g}$ is a connected, orientable surface of genus $g$, where $2 g+2=h$, and where $f^{\prime}\left(F_{g}\right)$ lies in $T^{2} \subset T^{3}$ up to homotopy. It follows that $f_{*}^{\prime}\left[F_{g}\right] \neq 0$ in $T^{2}$ and $T^{3}$ since this homology class mod 2 agrees with $f_{*}\left[U_{h}\right]_{2}$.

It is clear that $f_{*}^{\prime}\left(H_{1}\left(F_{g}\right)\right)$ is a rank 2 summand, since it is of rank at most 2 and adding a single generator yields all of $H_{1}\left(T^{3}\right)$. If we could be sure that $f_{*}^{\prime}\left[F_{g}\right]$ is a primitive homology class, then by Theorem $2.4, f^{\prime} \simeq h^{\prime}$, where $h^{\prime}: F_{g} \rightarrow T^{3}$ is an embedding onto a nonseparating surface. But it appears that we need to work a little harder for this in general.

Now consider the base case of our induction, when $h=4$. Then $g=1$. In this case it does follow that $f_{*}^{\prime}\left[F_{1}\right]$ is a primitive homology class, because for maps of the torus the primitivity and rank 2 summand conditions coincide. (In this case the unimodularity condition is automatically satisfied.) Thus $f^{\prime} \simeq h^{\prime}$, where $h^{\prime}: F_{1} \rightarrow T^{3}$ is an embedding onto a subtorus. Now $f$ is obtained from the embedding $h^{\prime}$ by running a tube along an arc from one side of $h^{\prime}\left(F_{1}\right)$ to the opposite side, since $U_{h}$ itself is nonorientable.

The condition that $f_{*}$ is onto implies that this arc goes just once around up to homotopy. Therefore one can homotope the arc so that it is embedded and meets $h^{\prime}\left(F^{\prime}\right)$ only in its end points. It follows that the result of tubing along the arc produces the desired embedded nonorientable surface.

Now henceforth inductively assume that $h \geq 6$ and is even. 
Given that the kernel $K$ is unimodular, there is a pair of orientation preserving homology classes $\{\alpha, \beta\}$ in the kernel such that $\alpha \cdot \beta=1$. One can then represent such a hyperbolic plane in $K$ by a pair of two-sided simple closed curves $\{A, B\}$ meeting transversely in a single point in the capped-off orientable surface containing a subsurface of $U_{h}$ carrying $K$. (Compare the proof of Proposition 4.2 in Edmonds [3] or Proposition 3.13 below, which we include for the reader's convenience.) Then one simultaneously surgers away the pair $\{A, B\}$, by removing a neighborhood of the union $A \cup B$, which is a punctured torus, and replacing it with a disk. The result is a surface $U_{h-2}$ and a map $f^{\prime \prime}: U_{h-2} \rightarrow T^{3}$ that inherits all hypothesized properties of $f: U_{h} \rightarrow T^{3}$. By induction $f^{\prime \prime}$ is homotopic to an embedding. One then recreates the original surface and an embedding homotopic to the original map $f$ by adding a small trivial handle.

Finally, we include, for the convenience of the reader, a proof of the realizability of homology classes of intersection number 1 , as used in the preceding theorem.

Proposition 3.13. Let $\alpha, \beta$ be two homology classes in $H_{1}(F)$ where $F$ is a closed orientable surface, with intersection number $\alpha \cdot \beta= \pm 1$. Then $\alpha$ and $\beta$ are represented by simple closed curves $A$ and $B$ meeting transversely in a single point.

Proof. The intersection number condition implies that $\alpha$ and $\beta$ are primitive classes. By [1], [8], or [9] there are simple closed curves $A$ and $B_{1}$ representing $\alpha$ and $\beta$ and meeting transversely in isolated points. By surgering $B_{1}$ at adjacent points of intersection on $A$ where $B_{1}$ crosses $A$ in opposite directions, we convert $B_{1}$ to a disjoint union $B_{2}$ of simple closed curves representing $\beta$ but meeting $A$ in just one point. Since $A$ does not separate $F$ we may tube together the components of $B_{2}$ in a way compatible with orientations on the components using paths in the complement of $A$. The result is a closed, but not necessarily embedded, curve $B_{3}$ representing $\beta$ and meeting $A$ in just one point. We may assume by a small perturbation if necessary that the self-intersections of $B_{3}$ are transverse. Slide the points of self-intersection along $B_{3}$ until they are near the point where $B_{3}$ meets $A$, together on the same side of $A$. These points of intersection can be eliminated by replacing small arcs of $B_{3}$ with arcs that go around the other side of $A$. The result is a simple closed curve $B_{4}$ meeting $A$ transversely in a single point and representing a homology class of the form $\beta+k \alpha$ for some integer $k$. For applications this suffices. But with more care we can change $k$ to 0 . Introduce $|k|$ small kinks into $B_{4}$ of the appropriate sign, without changing the homology class of $B_{4}$. Then eliminate these intersections by the same process, sliding arcs over $A$. The final result is the desired simple closed curve $B$. 
A more algebraic proof would argue that the pair $\alpha, \beta$ can be moved to a standard symplectic pair $a, b$ (having the desired representation by simple closed curves) by a symplectic automorphism of $H_{1}(F)$ which can be realized by a homeomorphism.

3.7. Examples. Here we give examples showing that each of the necessary conditions (4) and (5) in the Embedding Theorem 3.12 is independent of the other and of the other conditions for maps to $T^{3}$. It does not make sense to treat condition (2) as being independent, since it is required for formulating condition (5). We will also prove that condition (3) follows from the other conditions, including the unimodularity condition.

EXAMPle 1. Here we give an example of an immersion of a nonorientable surface into $T^{3}$ that satisfies conditions (1)-(4) of the Embedding Theorem 3.12, but for which the intersection pairing on the kernel $K$ is not unimodular.

A minimal example will be of the form $U_{6} \rightarrow T^{3}$. Start with the standard embedding $T^{2} \rightarrow T^{3}$ onto the subtorus corresponding to the first two coordinates. Extend this to an immersion of $U_{4}=T^{2} \# U_{2}$ by adding a nonorientable handle as the boundary of a neighborhood of an arc that runs three times around the third factor $* \times * \times S^{1}$ of $T^{3}$. By going an odd number of times around we ensure that $w_{1}\left(U_{h}\right) \in f^{*} H^{1}\left(T^{3} ; \mathbb{Z}_{2}\right)$.

Then add an orientable handle as the boundary of a neighborhood of an arc that runs twice around the third factor $* \times * \times S^{1}$ of $T^{3}$. By going an even number of times around we ensure that still $w_{1}\left(U_{h}\right) \in f^{*} H^{1}\left(T^{3} ; \mathbb{Z}_{2}\right)$. By going a number of times around that is relatively prime to the number used for the nonorientable handle, we guarantee that the corresponding immersion $U_{6}=T^{2} \# U_{2} \# T^{2} \rightarrow T^{3}$ is surjective on homology.

Let $H_{1}\left(U_{6}\right)$ have standard generators $a_{1}, b_{1}, u, v, a_{2}, b_{2}$, with respect to the above connected sum decomposition, where $u$ is orientation-reversing and $v$ has order 2 . Let $H_{1}\left(T^{3}\right)$ have a standard basis $x, y, z$ corresponding to the expression as a product of circles. The induced homomorphism $H_{1}\left(U_{6}\right) /$ torsion $\rightarrow H_{1}\left(T^{3}\right)$ is then given, with respect to the bases $a_{1}, b_{1}, u$, $a_{2}, b_{2}$ and $x, y, z$, by the matrix

$$
\left[\begin{array}{lllll}
1 & 0 & 0 & 0 & 0 \\
0 & 1 & 0 & 0 & 0 \\
0 & 0 & 3 & 0 & 2
\end{array}\right] .
$$

The kernel has a basis consisting of $a_{2}$ and $2 u-3 b_{2}$. The matrix of intersections is 


$$
\left[\begin{array}{cc}
0 & -3 \\
3 & 0
\end{array}\right]
$$

We conclude that this map is not homotopic to an embedding.

ExAmple 2. Next we give an example of an immersion of a nonorientable surface into $T^{3}$ satisfying conditions (1)-(3) and (5) but not (4) of the Embedding Theorem 3.12, so that it does not induce a surjection on first homology. Let $f: U_{h} \rightarrow T^{3}$ be an embedding and let $p: T^{3} \rightarrow T^{3}$ be a nontrivial covering of odd degree. Then the composition $p f: U_{h} \rightarrow T^{3}$ satisfies the requirements.

Proposition 3.14. A map of a nonorientable surface into $T^{3}$ satisfying conditions (1)-(2) and (4)-(5) of the Embedding Theorem 3.12 must also satisfy condition (3), i.e. that the mod 2 homology class has $f_{*}\left[U_{h}\right]_{2} \neq 0$.

Proof. As in the proof of the Embedding Theorem one may surger away the kernel/torsion of a map $f: U_{h} \rightarrow T^{3}$ satisfying the hypotheses (1)-(2) and (4)-(5). The result is necessarily a map $f^{\prime}: U_{4} \rightarrow T^{3}$, satisfying the same hypotheses, but where the kernel/torsion is now 0 . Next one may surger the map along a simple closed curve representing a homology class of order 2, Poincaré dual to the first Stiefel-Whitney class. The result is a map $f^{\prime \prime}: T^{2} \rightarrow T^{3}$. The image of this map on $H_{1}$ must be a rank 2 summand, since adding a single generator to the image yields a surjection. It follows that $f^{\prime \prime}$ is homotopic to an embedding onto a standard subtorus. In particular, $f^{\prime \prime}$, and hence $f$, has the property that the image of the mod 2 fundamental class of the surface is nontrivial, as required.

\section{References}

[1] D. Bennequin, Sur les représentants lisses des classes d'homologie entière de codimension 1 des variétés, C. R. Acad. Sci. Paris Sér. A-B 285 (1977), A207-A210.

[2] G. E. Bredon and J. W. Wood, Non-orientable surfaces in orientable 3-manifolds, Invent. Math. 7 (1969), 83-110.

[3] A. L. Edmonds, Systems of simple closed curves on a surface, Enseign. Math. 42 (1996), 311-339.

[4] - Deformation of maps to branched coverings in dimension two, Ann. of Math. (2) 110 (1979), 113-125.

[5] W. End, Nonorientable surfaces in 3-manifolds, Arch. Math. (Basel) 59 (1992), 173-185.

[6] J. Hempel, 3-Manifolds, Ann. of Math. Stud. 86, Princeton Univ. Press, 1976.

[7] W. Jaco, Surfaces embedded in $M^{2} \times S^{1}$, Canad. J. Math. 22 (1970), 553-568.

[8] W. H. Meeks III and J. Patrusky, Representing homology classes by embedded circles on a compact surface, Illinois J. Math. 22 (1978), 262-269.

[9] M. D. Meyerson, Representing homology classes of closed orientable surfaces, Proc. Amer. Math. Soc. 61 (1976), 81-82. 
A. L. Edmonds

[10] J. W. Milnor and J. D. Stasheff, Characteristic Classes, Ann. of Math. Stud. 76, Princeton Univ. Press, 1974.

[11] F. Waldhausen, On irreducible 3-manifolds which are sufficiently large, Ann. of Math. (2) 87 (1968), 56-88.

Department of Mathematics

Indiana University

Bloomington, IN 47405, U.S.A.

E-mail: edmonds@indiana.edu

Received 14 January 2005;

in revised form 25 January 2008 